\title{
Nordiques
}

38 | 2019

Le petit héros scandinave

\section{Bibi de Karin Michaëlis et sa rencontre avec la réalité : Hygge lifestyle expérimenté par les enfants?}

Davide Finco

\section{OpenEdition}

1 Journals

Édition électronique

URL : http://journals.openedition.org/nordiques/296

DOI : 10.4000/nordiques.296

ISSN : $2777-8479$

Éditeur :

Association Norden, Bibliothèque de Caen la mer

Édition imprimée

Date de publication : 1 novembre 2019

Pagination : 85-98

ISBN : 979-1-0959140-4-4

ISSN : $1761-7677$

Référence électronique

Davide Finco, «Bibi de Karin Michaëlis et sa rencontre avec la réalité : Hygge lifestyle expérimenté par les enfants? ", Nordiques [En ligne], 38 | 2019, mis en ligne le 31 octobre 2020, consulté le 15 mars 2021. URL : http://journals.openedition.org/nordiques/296 ; DOI : https://doi.org/10.4000/nordiques 296

Nordiques 


\section{Bibi de Karin Michaëlis et sa rencontre avec la réalité : Hygge lifestyle expérimenté par les enfants?}

Davide Finco*

\section{RÉSUMÉ}

Le mot danois hygge, dont la définition a fait l'objet d'articles scientifiques et d'ouvrages de diffusion, est aujourd'hui l'un des termes qui caractérisent le plus fréquemment la culture et la perspective danoises sur ce monde. Dans de nombreux livres et articles (de plus en plus au cours des dernières années), ce mot est utilisé pour désigner un système de valeurs impliquant tout aspect de la vie quotidienne. Il est présenté comme étant le secret du bonheur danois, un état de bien-être confirmé par les enquêtes internationales depuis les années 1970. Dans The Danish Way of Parenting ${ }^{1}$ (2014), Jessica Alexander et Iben Sandahl examinent les différents aspects de ce mode de vie dans l'éducation des enfants. Les mots-clés utilisés par les auteurs et leurs champs d'application trouvent plusieurs correspondances significatives dans la vie quotidienne de Bibi, l'hérö̈ne de Karin Michaëlis. La différence réside dans l'attention aux expériences de la jeune fille dans la série de Michaëlis (premier livre de 1927, 1929). Cette contribution vise à évaluer dans quelle mesure Bibi illustre un mode de vie et un système de valeurs qui ont été formalisés par la suite dans le style de vie du hygge danois (ou mieux, dans la manière de devenir parents). On offrira ainsi une nouvelle perspective pour considérer Michaëlis comme le plaidoyer des droits de l'enfance et de ses espaces dans ses ceuvres littéraires.

\section{ABSTRACT}

The Danish word hygge, whose definition is the subject of scientific papers and dissemination works, is nowadays one of the terms that most frequently characterize Danish culture and world view. In many books and articles (more and more in the last few years), this word is used to label a value system involving any aspect of daily life, and is presented as the secret of Danish happiness, a state of wellness which international surveys have confirmed at least since 1970's. In The Danish Way of Parenting (2014), Jessica Alexander and Iben Sandahl investigate the different aspects of this way of living when upbringing children. The key words the authors use and their fields of application find several meaningful correspondences in Karin Michaëlis' Bibi's daily life, with the substantial difference that in Michaëlis' series (first book in 1927, 1929), the focus lies on the girl herself, who often reports her experiences. My contribution aims to assess to what extent Bibi exemplifies a way of life and a value system which later has been formalized in the nowadays worldwide discussed Danish hygge lifestyle (or, better, in its way of parenting), in this way offering a new perspective to consider Michaëlis' plea for children's rights and spaces in her literary.

* Davide Finco est maître de conférences en études scandinaves à l'université de Gênes. Sa thèse de doctorat portait sur la littérature de jeunesse en Scandinavie et en Italie au XXe siècle. Il explore actuellement le rôle des espaces urbains dans la littérature scandinave depuis la fin du XIX siècle et la réception de la littérature scandinave en Italie au cours du XXe siècle. Il a rejoint un groupe de recherche international (coordonné par le Centre d'études scandinaves de Lund-Copenhague) sur la " Nordic World Literature » et un groupe de recherche national sur les travaux d'August Strindberg.

1 Jessica Joelle Alexander, Iben Dissing Sandahl, The Danish Way of Parenting. What the Happiest People in the World Know about Raising Confident, Capable Kids, London, Piatkus, 2016 [2014]. 


\section{INTRODUCTION : HYGGE ET LITTÉRATURE POUR ENFANTS ?}

Comme annoncé dans le titre de la contribution, le sujet de cette étude pourrait paraître, dans le meilleur des cas, très à la mode ou bien vraiment tendancieux, voire envisagé, dans le pire des cas, comme une expérience risquée. En effet, le hygge lifestyle est devenu une sorte de marque nationale danoise au cours des dernières décennies, avec la publication de plusieurs travaux sur cette thématique, une littérature populaire facile à trouver dans les magasins de souvenirs ou à l'aéroport. D'ailleurs, selon certains spécialistes, ce phénomène aurait une origine britannique évidente et il aurait fini par influencer la manière dont les Danois se perçoivent, quand bien même cela semble artificiel ${ }^{2}$.

Quelle que ce soit la façon dont on l'aborde, certaines indications sont probablement nécessaires avant de développer notre sujet. Tout d'abord, cette contribution n'a pas pour but de présenter Karin Michaëlis, ou du moins son personnage le plus aimé, Bibi, comme la promotrice explicite d'une présumée philosophie du hygge. En même temps, les dimensions du hygge ne sont pas considérées ici comme les aspects les plus pertinents de son travail, même si sa focalisation sur une enfance particulièrement libre et engagée apparaît comme le message le plus significatif de son œuvre, de manière à faire de Bibi l'un des petits héros scandinaves que les articles de ce volume visent à étudier.

D'après les résultats de mes recherches, aucun livre sur le hygge ne mentionne Karin Michaëlis et, dans la plupart des cas, ils ne font pas non plus référence spécifiquement à la littérature danoise, car ils envisagent plutôt la " philosophie du hygge " comme une sagesse innée des Danois. Néanmoins, si nous nous concentrons sur Michaëlis et son personnage spirituel et vivant, une partie de la philosophie du hygge attribuée aux Danois peut nous aider à mieux souligner les caractéristiques pédagogiques pertinentes (et révolutionnaires) illustrées par les aventures de Bibi, les paroles de Bibi dans ses lettres et les commentaires du narrateur. Vue sous cet angle, ma tentative vise à présenter sous un jour différent la contribution de Michaëlis au renouveau de la littérature et à relier - autant que possible - deux phénomènes littéraires, tous deux profondément ancrés dans la culture danoise.

Le mot hygge ne couvre pas exactement l'ensemble des valeurs et des idées que je traiterai. Cependant, il peut être mis en relation avec chacune d'elles. Dans

2 Je me réfere en particulier à une communication du programme de la dernière conférence de l'IASS à Copenhague (août 2018) : Malene Breunig, « Danish hygge » - A fairy tale made in Britain, available on https://nors.ku.dk/english/calendar/2018/scandinavian-exceptionalisms/IASS2018_Abstracts.pdf [dernière consultation le 15.01.2019]. 
The Danish Way of Parenting ${ }^{3}$, ce concept mérite un chapitre spécifique, puisque le hygge devrait être un élément nécessaire d'une pratique beaucoup plus large de la vie quotidienne auprès des enfants. Si nous le considérons, d'une part, comme un état de bien-être existentiel pouvant être poursuivi par une éducation appropriée, et, d'autre part, la manière dont cela peut être transmis aux enfants, nous nous rendons compte à quel point des valeurs fortes telles que la liberté et le respect peuvent former de futurs adultes conscients, capables d'un style de vie conforme à la philosophie du hygge. La preuve en est que vers la fin de leur œuvre, Alexander et Sandahl rappellent le lien qu'ils ont souligné entre hygge et éducation :

Qu'est-ce qui a fait que les Danois se maintiennent au sommet de l'échelle $\mathrm{du}$ bonheur pendant si longtemps ? Comme nous l'avons vu [...], c'est très simplement la manière dont ils élèvent leurs enfants. C'est un héritage qui perdure, se transmet à travers les générations, en formant des adultes sûrs d'eux-mêmes, confiants, équilibrés, résilients et heureux ${ }^{4}$.

Cette brève description, qui résume la plupart des analyses et des expériences présentes dans le manuel, convient presque parfaitement au personnage de Bibi - comme nous allons le voir - en particulier si nous considérons ses aventures et le développement de sa personnalité dans le cadre d'un processus plus long, comme beaucoup de lecteurs et d'experts l'ont bien compris : un chemin vers la libération féminine qui part de l'enfance. Il ne faut ainsi pas oublier que Bibi a un double statut de "minorité » : en tant qu'enfant mais aussi en tant que petite fille. Dans son émancipation provocatrice au regard d'une éducation traditionnelle, elle réalise à la fois les rêves (et les besoins) des enfants et les revendications de la libération féminine. De manière générale, le renouvellement de la littérature pour enfants a été à plusieurs reprises lié à la lutte pour les droits des femmes. Chez Michaëlis, nous pouvons observer que l'auteure a longtemps examiné et de manière approfondie la condition matérielle et morale des femmes adultes avant de présenter les aventures de Bibi5

3 Jessica Joelle Alexander, Iben Dissing Sandahl, op. cit.

4 "What is it that has kept the Danes at the top of the happiness charts for so long? As we have seen [...], it is, quite simply, the way they raise their children. It is a legacy that continues on, repeating itself through the generations, resulting in self-assured, confident, centered, happy, and resilient adults. "Toutes les références en note concernent Jessica Joelle Alexander, Iben Dissing Sandahl, The Danish Way of Parenting, London, Piatkus, 2016, p. 143. Traduction de la rédaction.

5 Voir Karen Klitgaard Povlsen, "Karin Michälis ", in Dansk Kvindebiografisk Leksikon, 2003, disponible sur : http://www.kvinfo.dk/side/170/bio/1437/query/michaelis [dernière consultation le 14.1.2019] ; Donatella Ziliotto, "Influenze della letteratura Nordica. Dahl, Michaëlis, Lindgren, Jansson », LG Argomenti, vol. XL, n. 3, janvier-février 2004, p. 24. 


\section{ROMAN ET MANUEL : QUELQUES REMARQUES GÉNÉRALES}

Dans la présente analyse, je me réfèrerai exclusivement au premier livre de la série, à savoir Bibi. En lille Piges Liv (Bibi, une petite Danoise) $)^{6}$, publiée aux États-Unis (où se trouvait Michaëlis) en 1927 et au Danemark en 1929. Son héroïne est une enfant de onze ans - dont l'âge n'est toutefois révélé aux lecteurs que par ses grands-parents vers la fin du livre. Elle est la fille d'un chef de gare, orpheline de mère et, grâce au travail de son père, elle est autorisée à voyager gratuitement en train à travers le Danemark. En outre, et surtout, Bibi jouit d'une grande liberté ; son père lui accordant une grande confiance et la laissant partir quand elle le souhaite, à condition qu'elle le tienne informé de ses voyages et de ses ballades. La jeune fille est animée par la curiosité, le sens de l'aventure, l'esprit d'initiative et une grande capacité à s'adapter à la plus grande variété de situations. Son pragmatisme s'avère être assez inhabituel pour une enfant de son âge et ses rencontres étranges sont rapportées dans des lettres enjouées écrites à son père, pleines d'humour, souvent involontairement, en raison de ses fautes d'orthographe et de syntaxe, et enrichies par ses propres dessins.

La série Bibi joue un rôle important dans la littérature de jeunesse scandinave qui a marqué un tournant dans la tradition du Nord et a eu une certaine influence sur les écrivains italiens pour enfants : l'écriture de Michaëlis est vive, ironique et amusante, avec beaucoup d'empathie pour le point de vue et le langage des enfants, et ces qualités, associées aux caractéristiques sans précédent de l'héroïne, ont déterminé l'impact considérable que ses livres ont eu sur l'enfance de certains auteurs d'œuvres pour la jeunesse ${ }^{7}$. Dès la première lecture, ces histoires montrent qu'elles se fondent sur une position pédagogique claire, ce qui nous permet d'identifier plusieurs similitudes entre les principes, les résultats et les arguments du manuel et le roman de Bibi. Ceci révèle que cette perspective littéraire pourrait être perçue comme une sorte d'illustration implicite de nombreux conseils donnés aux parents dans le manuel. Nous avons été frappés par le fait qu'Alexander et Sandahl ne présentent pas leur méthode comme le résultat d'acquisitions modernes, mais plutôt comme une formalisation moderne - étayée par un grand nombre d'études scientifiques, qu'ils mentionnent - d'anciennes pratiques parentales profondément liées à la vision du monde danois. Ce type de marketing visait évidemment à rendre le livre plus attrayant (ou plus fiable grâce

6 Karin Michaëlis, Bibi. En lille Piges Liv, København, Jespersen og Pios Forlag, 1934 [1929].

7 Cf Davide Finco, «Freedom starts as early as childhood. Scandinavian children's (female) writers' impact on Italian children's literature before and after the Second World War ", in Robert Zola Christensen (ed.), A collection of articles based on presentation held at the CSS Conference 2017 - "Rethinking Scandinavia" (web proceedings), 2018, Lund, International web community for Scandinavian Studies, https://csspublications.net/konf17 proceedings/2018/2/16/freedom-starts-as-early-as-childhood [dernière consultation le 15.01.2019]. 
à la référence à la sagesse de toute une nation), mais il offre aussi un cadre bien défini pour répondre à mon hypothèse et évaluer le parallélisme entre les deux modes de narration.

De manière générale, on peut penser que la vie de Bibi est une sorte de grande ode au Danemark - comme le montre clairement l'introduction vive et ironique ${ }^{8}$. Le Danemark est défini comme un pays aussi petit qu'un très jeune enfant (nous pourrions imaginer que ce pays soit un enfant parmi les adultes et donc une forme de métaphore de la condition de Bibi elle-même). Il s’agit d'une célébration de la liberté, conquise kilomètre par kilomètre, balade après balade, rencontre après rencontre : cette représentation construit l'image d'un monde beaucoup moins dangereux que celle que beaucoup d'adultes inquiets mobilisent pour le raconter à leurs enfants : un monde riche en opportunités, où s'accepter soi-même, reconnaître ses sentiments et faire preuve d'une grande ouverture d'esprit pour éliminer les préjugés sont des outils fondamentaux, aussi bien pour les enfants que pour les adultes ; l'ambition est de vivre une expérience de vie pleine et authentique, d'apprécier et développer ses côtés agréables, tout en se confrontant à ses aspects plus désagréables.

Tous ces éléments sont présentés et précisés dans le manuel d'Alexander et Sandahl, et pourtant, ils ne mentionnent pas Karin Michaëlis, ni même des œuvres littéraires, mais plutôt certaines expériences, études scientifiques et monographies spécialisées. Jessica Alexander est américaine et, $s$ 'inspirant de l'approche éducative de son mari, danois, elle s'est intéressée à ces sujets après la naissance de leur premier enfant et ce, malgré son très faible instinct maternel ${ }^{9}$. Iben Sandahl est, quant à lui, un psychothérapeute danois. Alexander raconte à quel point elle a toujours été impressionnée par la manière dont ses amis danois traitent leurs enfants et par les très bons résultats obtenus, alors que, reconnait-elle, la façon de faire est différente des pratiques nord-américaines courantes. Pour renforcer ses thèses, à de multiples reprises, elle établit une comparaison impitoyable entre les points de vue danois et états-uniens en matière d'éducation, de sorte que les positions américaines sont généralement décrites comme totalement à l'opposé du vertueux modèle danois.

Leur manuel a été élaboré après treize années d'observations et d'interviews et il a été écrit dans le but de sensibiliser des parents (ou de les aider) et leur faire prendre conscience des besoins réels des enfants ainsi que des carences ou des

8 Karin Michaëlis, op. cit., p. 7-19. Cette introduction était en quelque sorte nécessaire pour les lecteurs qui avaient une idée vague du Danemark. Dans le premier chapitre, nous retrouvons encore l'hérö̈ne en train de dessiner une carte du Danemark sur une fenêtre couverte de vapeur, comme si elle montrait les grandes limites de son monde.

9 Jessisca Alexander, Iben Sandahl, Danish Way of Parenting, op.cit., p. xix. 
erreurs les plus courantes dans le processus de parentalité. Cette structure fait du manuel une sorte d'équivalent du roman de Bibi, car, dans le livre de Michaëlis, c'est l'enfant qui parle et raconte ses propres expériences (même si le narrateur fait plusieurs commentaires), révélant ainsi indirectement l'éducation particulière qu'elle a reçue.

\section{DEUX FAÇONS DE CONCEVOIR LE POINT DE VUE DANOIS SUR LES ENFANTS}

Les principes de cette méthode danoise sont indiqués au début du manuel par l'utilisation de mots clés (dont l'acronyme compose le mot " parent " : play (jeu), authenticity (authenticité), reframing (recadrage), empathy (empathie), no ultimatum (pas d'ultimatum), togetherness (être ensemble) ${ }^{10}$. Le premier est le jeu (play) et les auteurs insistent sur l'importance pour les enfants de jouer en toute liberté et du temps perdu en apparence qui leur permet, au contraire, de beaucoup apprendre et d'oublier le stress quotidien. Bibi est devenue experte en la matière : elle sait très bien qu'elle trouvera des occasions de jouer partout et elle est consciente qu'elle sera capable de transformer les jeux en emploi, comme nous le verrons. Elle ne s'impose jamais un programme, car elle est totalement libre de gérer ses propres activités. Selon Alexander et Sandahl, la philosophie parentale danoise repose formellement sur le concept de "proximal development» (zone de développement proche) : c'est l'espace de liberté laissée (ou autorisée) aux enfants, une sorte d'amortisseur entre leurs actions et les actions de leurs parents. Il a été prouvé que cet espace est fondamental à tout âge pour le développement des enfants qui expérimentent ainsi l'autonomie ${ }^{11}$. Pour Bibi, cet espace est le Danemark entier (et, dans les romans suivants, parfois des lieux étrangers). Certes, elle dispose d'une liberté sans précédent et vit à une époque marquée par une organisation moins contraignante des activités quotidiennes - bien que non dénuée de tout contrôle social des enfants - si on établit la comparaison avec notre époque. Cependant, le roman pourrait entièrement être lu comme étant le compte rendu ou le résultat d'une expérimentation conduisant sans cesse à cet immense espace de développement proche, en fonction non seulement des expériences vécues, mais également des compétences acquises et perfectionnées.

10 Ibid., p. xi. Le hygge est mentionné dans la dernière catégorie : «Togetherness and hygge (coziness)». Dans le chapitre spécifique (p. 123), ce terme s'explique par " cozying around together ", ce qui évoque le fait de passer du bon temps, surtout à la maison avec des amis. Mais juste après (p. 124-125), en référence à l'étymologie du mot (de l'hyggja germanique: " penser ou se sentir satisfait »), ce concept acquiert le sens plus profond de "se sentir connecté aux autres".

11 Ibid., p. 16-17. Ce concept a été développé par le psychologue russe Lev Vigotskij : "His [Vigotskij's] thinking has, to this day, had a major influence on the scope of teaching in Danish schools ». Ibid., p. 152. Voir aussi : Leif Strandberg, Vigotskij i praksis, København, Akademisk Forlag, 2009. 
Une qualité humaine qui revient souvent dans le manuel, à la fois vis-à-vis des parents et des enfants, est la résilience (qui, tout comme le hygge, est devenue un mot à la mode). Terme tiré du domaine scientifique, qui désigne techniquement la capacité d'une substance ou d'un objet à reprendre forme (une sorte d'élasticité) est dans un sens large et commun, l'aptitude à s'adapter aux circonstances extérieures et à se relever de difficultés. Bibi montre qu'elle est vraiment "résiliente " - bien qu'elle ne soit jamais décrite avec ce mot - à plusieurs reprises, comme si la liberté totale dont elle profite l'avait définitivement amenée à développer cette capacité. Si nécessaire, elle est prête à inventer des histoires pour cacher sa véritable identité face à des inconnus ou survivre longtemps loin de chez elle en monnayant ses dessins ou en ramassant des objets d'occasion pour les revendre, ou bien encore à divertir les gens (et être payée) pour ses acrobaties.

À ce sujet, nous rappelons deux détails significatifs pour notre étude : tout d'abord, Bibi est vraiment douée pour le dessin mais beaucoup moins pour l'écriture. Cet élément est indiqué et commenté par le narrateur depuis le début du roman, avec le chaleureux conseil d'être patient et compréhensif face aux faiblesses des autres, car celui qui est brillant en quelque domaine l'est parfois moins dans d'autres ${ }^{12}$. De même, Alexander et Sandahl consacrent un chapitre entier à cet aspect, mis en relation avec le principe d'empathie, en désapprouvant fortement le climat de concurrence dans la société américaine exacerbé dès la petite enfance ${ }^{13}$. Ne pas exalter les mérites de ses propres enfants et ne jamais dénigrer ou minimiser les représentations ou les prestations d'autres enfants - au contraire, toujours leur adresser des mots d'encouragement, du moins lorsque l'on parle en leur présence - est envisagé comme l'un des piliers de la méthode parentale danoise ${ }^{14}$. Un second détail important est le fait que Bibi a appris l'une des acrobaties les plus utiles et les plus appréciées (à savoir escalader un mur à mains nues) d'un cambrioleur, à qui elle avait sauvé la vie en suçant le poison d'une blessure provoquée par un serpent. Le cambrioleur se rend ensuite aux mains de la justice, mais il reste l'ami de Bibi et de son père, ce qui constitue un élargissement du monde de l'enfant au royaume du crime et prouve que quelque chose de positif peut venir de n'importe qui. ${ }^{15}$ Etre ouvert aux autres sans préjugés et être prêt à déceler le côté positif de chaque situation et de chaque personne est une autre valeur qui est généralement transmise par les parents danois à leurs enfants, selon les auteurs.

12 Karin Michaëlis, op. cit., p. 38.

13 Jessica Alexander, Iben Sandahl, op. cit., p. 77-78.

14 Ibid., p. 36.

15 Karin Michaëlis, op. cit., p. 63-68. 
En faisant écho aux théories psychanalytiques, Alexander et Sandahl introduisent le concept d'internal compass (compas intérieur), mieux exprimé dans la définition d' "Internal vs. External Locus of Control " (validation interne ou externe) : ces catégories désignent les personnes qui croient et ressentent un sentiment général de contrôle sur leur propre vie, et les personnes qui estiment que leur vie est principalement façonnée par les autres (ou par des circonstances extérieures). En outre, au cours des cinquante dernières années, les auteurs dénoncent l'augmentation du nombre d'individus ayant un external locus of control, c'est-àdire une méfiance générale à l'égard de leurs propres capacités, en particulier chez les enfants ${ }^{16}$. Bibi est un exemple clair et fort d'une personne qui veut créer sa propre existence en explorant toute sa liberté et le monde entier, bien au-delà de sa maison et de sa ville.

Elle contrôle sa vie même dans le choix de son nom, une chance qui est refusée aux enfants quand ils sont trop petits : en fait, elle porte le nom de sa mère décédée, Ulrikke Elisabeth, dite Ulla, mais elle l'échange avec le nom d'une amie obtenu après une sorte de négociation commerciale : à ce moment Bibi demande au crieur des villes une communication officielle de cet important changement ${ }^{17}$. Plus tard, elle se rend sur la tombe de sa mère - et ce, malgré l'interdiction de son père - et recherche des informations à son sujet en prenant contact avec la nourrice de celle-ci. L'optimisme réaliste de Bibi (realistic optimism est une autre catégorie utilisée par Alexander et Sandahl ${ }^{18}$ ) ne capitule jamais : elle est capable de faire correspondre l'insouciance typique de l'enfance au courage de poursuivre ses propres objectifs, et elle ne devient inquiète que face à l'imminence d'un danger.

Comme nous l'avons indiqué auparavant, dans le roman, nous ne lisons pas seulement ce que fait Bibi, mais aussi ce qu'elle écrit à ce sujet. C'est un point crucial. La langue écrite est complexe et difficile pour un enfant. De plus, c'est l'outil officiel de la communication que Bibi est en train d'assimiler à l'école, mais elle prend la liberté de faire des comptes rendus aussi vivants que possible, par le biais d'erreurs et d'associations amusantes dans sa syntaxe maladroite. En d'autres termes, elle réélabore ses expériences dans ses lettres, ce qui permet au lecteur de saisir son point de vue et de jouer avec la réalité en soulignant ses aspects les plus comiques ou les plus étranges. Ce travail tant spontané que nécessaire (car Bibi doit à la fois s'exprimer et informer son père quand elle est loin) compose une structure originale entre fiction et monde réel où les expériences vécues sont

16 Jessica Alexander, Iben Sandahl, op. cit., p. 15-16.

17 Il s'agit de sa première action dans le roman qui est le sujet du deuxième chapitre, après la longue et riche représentation du Danemark. Cf Karin Michaëlis, op. cit., p. 30-36.

18 Jessica Alexander, Iben Sandahl, op. cit., p. 51-54. Voir aussi : Ho Cheung William Li, Oi Kwan Joyce Chung, "The Relationship Between Children's Locus of Control and Their Anticipatory Anxiety ", Public Health Nursing 26, n. 2, 2009, p. 153-160. 
en quelque sorte reconsidérées au prisme de l'imaginaire. De cette manière, on peut mieux comprendre la réalité inexprimable des enfants, mettre les drames en perspective, tandis que les bonnes rencontres sont réévaluées, montrant ainsi que toute place dans le grand monde offre des opportunités d'ouverture vers les personnes sensibles. Cette capacité correspond très bien à ce qu'Alexander et Sandahl définissent comme un reframing qui reformule les événements, les sentiments et les états d'esprit pour ne pas être submergé par la réalité et pour surmonter les traumatismes ${ }^{19}$. Le reframing est une attitude constituant l'un des piliers de la méthode parentale danoise et qui a pour but d'apprendre aux enfants à réagir quand ils pourraient être accablés, en mettant les situations en perspective. En réalité, encore une fois, nous ne savons rien de ce que le père de Bibi lui a expliqué et enseigné au cours des années précédentes et pourtant, nous pouvons très facilement évaluer l'influence de son éducation et de son exemple.

Si nous restons dans la dimension de la relation père-fille, l'un des principes que nous pouvons qualifier de règle formalisée et qui guide la vie libre et souvent désordonnée de Bibi est la "parole d'honneur». Un chapitre du roman présente le "petit ", le mot d'honneur ordinaire généralement correct et fiable (même si certaines personnes n'en n'ont pas), et le " grand » que Bibi réserve à son père, celui qui a le droit d'être informé du parcours de Bibi par des messages qu'elle laisse avant de partir et de ses lettres : sa grande confiance en sa fille est nourrie par ce contact permanent ${ }^{20}$. Cette règle est si importante pour Bibi qu'elle se rend toujours compte quand elle risque de la rompre (et elle ne le fait qu'en cas de nécessité extrême) et elle réfléchit toujours à la manière de se comporter et d'agir en vertu de cette norme. Dans la grande liberté qu'il a laissée à sa fille, le père de Bibi a quelque peu renégocié les règles de base d'une éducation traditionnelle, tout en trouvant un compromis lui permettant néanmoins de se sentir en sécurité malgré l'instabilité généralisée de la vie ${ }^{21}$. Ce résultat est l'un des objectifs de la méthode danoise proposée par Alexander et Sandahl, qui pensent que les enfants doivent comprendre le sens des devoirs qui leur ont été imposés et que les parents peuvent toujours (et devraient) en parler avec eux ${ }^{22}$. Même dans ce cas, le lecteur n'assiste pas aux dialogues entre Bibi et son père au cours des années, mais réalise à quel point Bibi a compris les règles principales d'une existence basée sur la confiance mutuelle.

19 Ibid., p. 54-55 et 70-71. Voir aussi : Michael White, Narrativ teori, København, Hans Reitzels Forlag, 2006 [1995] ; Alice Morgan, Narrative samtaler, København, Hans Reitzels Forlag, 2005.

20 Karin Michaëlis, op.cit., p. 57-59. «Papsen SKAL HAVE SIT BREV» (Papounet VA AVOIR SA LETTRE) (Ibid., p. 61). Ce n'est que le sommet d'une série d'attitudes bienveillantes que Bibi montre pour les autres : elle sait comment demander gentiment de la nourriture ou des boissons, et où elle peut trouver un refuge loin de chez elle.

21 Jessica Alexander, Iben Sandahl, op. cit., p. 38-43.

22 Ibid. 
Selon les auteurs du manuel, l'une des erreurs (et des dangers) les plus récurrents dans la pratique parentale consiste à étiqueter les enfants (label children), en les décrivant comme mauvais, paresseux ou incapables, en raison de leur attitude dans des circonstances spécifiques. Cela cause de l'angoisse et de la frustration chez les enfants et est, en outre, profondément injuste, dans la mesure où le comportement des enfants à un moment précis peut être lié à des raisons fortuites (faim, fatigue, distraction, etc.), mais cela n'affecte en rien leurs qualités intrinsèques ${ }^{23}$. Les auteurs encouragent donc les parents à limiter les réprimandes. Comme dans un exercice rhétorique, ils donnent de nombreux exemples, suivant le principe $\mathrm{du}$ " there are no bad children, just bad behaviours", un précepte bien connu des parents danois. Apparemment, nous n'avons rien de semblable dans le roman car toute l'action est centrée sur l'héroïne et ses paroles. Cependant, il $\mathrm{y}$ a des passages où nous voyons concrètement cette " erreur d'étiquetage ». Un des exemples concerne la première rencontre de Bibi avec « le peuple gris ", c'està-dire ses nobles grands-parents maternels qui n’ont jamais pardonné à leur fille d'avoir épousé un chef de gare, une prétendue mésalliance, que Bibi considère, à juste titre d'ailleurs, comme la cause réelle du décès de sa mère. Le dialogue entre les grands-parents et la jeune fille est très rude : Bibi raconte fièrement son quotidien au couple scandalisé et choqué par la vie sauvage et extravagante menée par leur petite-fille. Cette dernière affirme ardemment qu'elle n'a pas besoin de savoir comment elle doit se comporter (et, ironiquement elle le fait en rappelant son statut de comtesse !), tandis que les lecteurs peuvent évaluer à travers le roman que sa façon de faire lui permet de développer sa personnalité2 ${ }^{24}$.

Mais c'est surtout lors du onzième chapitre, intitulé Else Prast ${ }^{25}$, évoquant la rencontre de Bibi avec la nourrice de sa mère, que cette question est systématiquement examinée. Le père de Bibi reçoit trois lettres : la première - la plus longue autour de cette rencontre émouvante - relate tout ce qu'elle a appris de l'enfance de sa mère ; la deuxième est écrite par la directrice de l'école où Bibi est inscrite. Elle se plaint du manque de discipline de la jeune fille, tout en reconnaissant ses qualités potentielles, et y raconte par le menu détail tous les incidents que Bibi a causés à l'école ; enfin, la troisième est écrite par les nobles grands-parents qui, informés sur le mode de vie anticonformiste de leur petite-fille, affirment clairement leur volonté d'intervenir dans son éducation. Le père de Bibi répond à aux deux dernières lettres, employant plus de temps pour celle adressée aux grandsparents, car il saisit l'occasion pour leur répondre en détail, tentant de démontrer qu'il n'est pas, comme ceux-ci l'accusent, le père irresponsable d'une enfant mal éduquée. Cet échange écrit est le seul passage du roman où les voix et les positions

23 Ibid., p. 57-62.

24 Karin Michaëlis, op. cit., p. 77-81.

25 Ibid., p. 125-148. 
des adultes passent au premier plan et où les lettres des adultes sont inévitablement mises en parallèle avec les nombreuses lettres de Bibi qui ont précédé. Cela évoque une sorte de dialogue idéal qui suit le moment où l'hérö̈ne a montré à quoi son monde ressemble.

Cette structure est un renversement de rôle manifeste en faveur de la petite héroïne, mais c'est aussi la tentative de déconstruction d'une éducation traditionnelle et de son discours : le père conseille amicalement à l'entourage familial de considérer le comportement et la situation de Bibi de manière objective ${ }^{26}$. Le roman ainsi que les analyses et recommandations du manuel présentent des correspondances évidentes. En se fondant sur les arguments du père de Bibi, le plaidoyer est d'établir une relation authentique et empathique avec les enfants sans trop prêter attention à ce que les autres pensent d'eux ou de vous ${ }^{27}$. Finalement, l'expulsion de Bibi de deux écoles la fait entrer en contact avec ses grandsparents : la splendeur de leur villa lui plait mais, à la toute fin du roman, elle réaffirme dans une lettre à son père que "jeg lar mig nu ikke addeptere ${ }^{28}$ " (je ne serai pas adaptée) par eux. Une confusion amusante entre les mots « adopter » et " adapter " qui résume néanmoins de manière très efficace le processus de contrôle des conduites enfantines par les adultes, qui passe par la catégorisation et l'utilisation d'étiquettes.

À mon avis, l'un des aspects les plus essentiels de la méthode parentale danoise est le principe d'authenticité, c'est-à-dire la nécessité d'être honnête avec les enfants, non seulement en leur demandant ce qu'ils peuvent réellement faire, d'expliquer pourquoi certaines interdictions ou obligations sont importantes, mais aussi en révélant ses propres fragilités, dans un échange mutuel vers une reconnaissance des sentiments des uns et des autres. Les adultes ne doivent pas perpétuer leurs "réglages par défaut" ("default settings») en imposant sans pitié des règles à leurs enfants : au contraire, ils doivent plutôt leur parler et développer leur capacité à reconnaitre leurs états d'esprit (et le fait que les sentiments sont des manifestations temporaires). Ce genre d'échange ne se produit jamais entre Bibi et les autres personnages. Pourtant, le narrateur commente plusieurs fois ses émotions et ses réflexions sur le fait qu'elles peuvent être incommunicables, alors que toutes sortes de sentiments, de la joie à la colère, de la peur à l'angoisse,

26 Ibid., p. 147-148.

27 Jessica Alexander, Iben Sandahl, op. cit., p. 111.

28 Le mot peut être considéré comme une erreur typique de Bibi pour qui " adopter " est un terme trop difficile, mais il est facile d'être considéré comme la crase d'" addere " (résumé) et " adaptere " (adapter). Karin Michaëlis, op. cit., p. 234. 
de l'enthousiasme à la frustration, sont légitimes car profondément humaines ${ }^{29}$. D'autre part, il existe un aspect éthique lié à cette authenticité, au sens moral du terme, et qui se développe plus tôt que prévu. Tout comme Alexander et Sandahl sont convaincus que les enfants peuvent beaucoup comprendre du comportement et des intentions des adultes, le narrateur du roman de Bibi assure que " nous le savons très bien - même si nous agissons comme si nous ne pouvions vraiment rien faire, car nous ne savions pas que quelque chose clochait. On sait toujours quand c'est faux $!^{30}$ ». Cette considération s'adresse à tous, adultes et enfants, et les place au même niveau, donnant un sens éthique même chez les enfants.

Contrairement à Fifi Brindacier qui incarne les rêves de pouvoir des enfants tout en développant un dialogue fructueux avec deux enfants " normaux " tels que Tommy et Annika, révélant ainsi ses propres compétences pédagogiques et son propre sens des responsabilité (en plus de défendre la cause des enfants), Bibi doit assumer à la fois le rôle d'enfant "normal » et d'enfant «spécial », sans l'appui d'un camarade du même âge, mais avec un grand désir de rentrer en contact avec le monde des adultes. En ce sens, Bibi incarne la curiosité des enfants plutôt que leur désir de pouvoir, et elle grandit tout au long de l'œuvre en montrant comment elle a acquis ses compétences et sa conscience éthique et morale. Bibi fait preuve d'une " mentalité de croissance " ("growth mind-set»), dans le sens où ses réalisations dépendent de son travail, et non "figée " ( fixed mind-set»), dans le sens où ses performances dépendent de ses qualités innées et immuables. Comme le lecteur l'aura peut-être deviné, ces catégories sont illustrées dans le manuel pour les parents et, d'après les auteurs, les parents danois savent bien comment favoriser cette première attitude ${ }^{31}$.

Les deux derniers aspects qu'il convient de mentionner sont le concept d'empathie qui est également implicite dans certaines de nos observations précédentes ainsi que la question du respect de la vie privée, ou plutôt de l'intimité. L'une des principales suggestions d'Alexander et Sandahl est de ne pas limiter les enfants aux expériences familiales mais d'élargir plutôt leurs contacts. À ce propos, nous pourrions peut-être exprimer cette idée avec le proverbe africain : "Il faut un village entier pour éduquer un seul enfant». Bibi semble porter cette logique

29 Cf Jessica Alexander, Iben Sandahl, op. cit., p. 6-8 ; Kenneth Rubin, Ock Boon Chung (ed.), Parental Beliefs. Parenting, and Child Development in Cross-Cultural Perspective, London, Psychology Press, 2013. Pour authenticity, voir : Jessica Alexander, Iben Sandahl, op. cit., p. 31-46.

30 Karin Michaëlis, op. cit., p. 52. «det ved man jo altid saa udmarket godt selv-ogsaa, hvis man lader, som om man 'virkelig ikke kunde gøre for det', for man vidste ikke, det var galt. Man ved altid, naar det er galt!»

31 Jessica Alexander, Iben Sandahl, op. cit., p. 37-38. Voir aussi : Carol S. Dweck, Mindset. The New Psychology of Success, New York, Random House, 2006, and Carol S. Dweck, Carol Dweck Revisits the "Growth Mindset ", 2015, accessible sur https://www.stem.org.uk/ system/files/communityresources/2016/06/DweckEducationWeek.pdf [consulté le 15.01.2019]. 
à l'extrême : son domicile n'est pas seulement au sein de sa famille, bien que son père soit toujours sa principale référence (d'ailleurs, son statut d'orpheline de mère, qui fait écho à celui de Pippi, répond de manière provocatrice aux tristes descriptions des orphelins des romans du XIX ${ }^{\mathrm{e}}$ siècle). Ce n'est pas non plus chez ces gens qu'elle connaît dans sa ville, mais bien chez ceux qu'elle rencontre à travers le Danemark.

En ce qui concerne le respect de l'intimité des enfants, qui leur conferre toute leur dignité, une leçon exemplaire est donnée lorsque le narrateur interrompt soudainement le récit après avoir fait de Bibi un compagnon d'aventures pour les lecteurs et après avoir décrit tous ses états d'âme : «Et maintenant, c'est comme quand on ferme les rideaux, on éteint la lampe et on se couche, parce que maintenant Bibi est complètement seule avec sa défunte mère et personne n'a le droit de savoir comment elles passent la nuit. ${ }^{32}$ " De cette manière, un adulte interfère comme pour prendre soin de la jeune protagoniste pourtant si indépendante.

Comme nous l'avons montré, il existe de nombreux points de contact entre la pratique pédagogique "danoise » telle qu'étudiée et reconstruite par Alexander et Sandahl et la caractérisation de la personnalité, des actions et du parcours éducatif de Bibi de Karin Michaëlis. Après cette analyse ils pourraient être formalisés par des mots-clés de la psychologie : du développement proche à la résilience, de l'autonomie à l'empathie, de l'authenticité à l'optimisme réaliste et à la mentalité de croissance (growth-mind set). De cette rapide enquête à la recherche de correspondances entre les textes, il ressort d'abord la clarté des valeurs pédagogiques (et sociopolitiques) des aventures de Bibi, comme autant de lecteurs l'ont expérimenté (et parmi eux de futurs écrivains pour enfants). Les effets attendus (et vérifiés) par Alexander et Sandahl lors de l'adoption de leur méthode, qui fait principalement référence aux contextes de l'éducation danoise traditionnelle, semblent être pertinents pour la petite héroïne et protagoniste du roman de Michaëlis.

Nous pourrions enfin mentionner que Michälis a également réfléchi à l'éducation des enfants dans une œuvre qui a précédé sa série Bibi - et qui pourrait être considérée comme une sorte de lien entre sa littérature pour adultes et ses livres pour enfants : il s'agit de l'essai Gladens Skole (1914). S'inspirant du Mädchenrealgymnasium, le projet éducatif d'un lycée pour filles fondé à Vienne par Eugenie Schwarzwald, Michaëlis aborde certaines questions concernant l'idée d'une éducation qui prend réellement en compte les rythmes et les besoins des enfants.

32 Karin Michä̈lis, op. cit., p. 111. "Og nu er det, som naar man ruller Gardinet ned, slukker Lampen og gaar i Seng, for nu er Bibi ganske alene med sin dode Mama, og det kommer ingen ved, hvordan Natten gaar for de to." 
Nous n'avons pas assez d'éléments pour affirmer que ces éléments proviennent véritablement d'un héritage culturel propre aux Danois. Seuls ceux-ci pourraient le confirmer ou y faire objection. Comme convenu, nous n'avons pas précisé toutes les dimension que peut revêtir la notion de hygge - à laquelle Alexander et Sandahl consacrent un chapitre rempli de suggestions pratiques - car, dans notre lecture, ceci est le point d'arrivée : développer la confiance des enfants, l'estime et la confiance en soi, ainsi que les valeurs de collaboration et de compréhension mutuelles qui sont les prémisses pour apprendre à profiter de la vie à chaque instant, sans nécessairement allumer des bougies pour prendre une tasse de thé sur des coussins moelleux, comme le suggère une vision quelque peu publicitaire du hygge dans nos sociétés contemporaines. 